\title{
Radiation Efficiency Improvement Method for Multifeed Circular Polarization Antenna Array with Mutual Coupling Effect
}

\author{
Zijian Xing, Ling Wang, Jianying Li, and Kun Wei \\ School of Electronics and Information, Northwestern Polytechnical University, Xian 710072, China \\ Correspondence should be addressed to Ling Wang; lingwang@nwpu.edu.cn
}

Received 20 January 2014; Revised 6 March 2014; Accepted 10 March 2014; Published 3 April 2014

Academic Editor: Shaoqiu Xiao

Copyright (C) 2014 Zijian Xing et al. This is an open access article distributed under the Creative Commons Attribution License, which permits unrestricted use, distribution, and reproduction in any medium, provided the original work is properly cited.

In the circular polarization antenna array, the input impedance, axial ratio, and radiation pattern of antenna patch will be changed. Compared with the performances of single antenna, the mutual coupling also led to the low efficiency of all antennas. A novel radiation efficiency elevation method of multifeed circular polarization (CP) antenna array is demonstrated in this paper, in order to solve the problem. To prove the concept of novel method, dual-feed antenna with stubs is developed and fabricated. According to the measured results, each antenna would increase in efficiency by approximate $1 \mathrm{~dB}$ by applying this novel design method. Therefore, this paper provides dramatically useful results of lessening the negative effect resulting in mutual coupling.

\section{Introduction}

Nowadays, circularly polarized microstrip patch antennas (MPAs) are one of the conventional antennas in the communication and radar systems [1-5]. The most important characteristics of these antennas are competing to Faraday rotation, reducing raindrops reflection effects, needing no estimation of the necessary orientation of the antenna according to the polarization of the received signals, duplicating the channel capacity, applying the positive and negative circular polarization, and reducing the fading effects.

According to feed methods, the MPAs can be divided into two kinds as the single feed and the multifeed $[6,7]$. The structure of single feed circularity polarization antenna is greatly simple, because it does not need feed network. However, axial ratio (AR) and impedance bandwidth of single feed antenna are extremely narrow and sensitive. Although multifeed MAPs are complex in structure, they are widely applied in antenna array, because the axial ratio of which is more robust [8-10]. In small antenna array, the input impedance of each component is different because of mutual coupling. The efficiency of each component plays an important role in the amplitude consistence of the whole array. The radiation pattern of each component is also different because it is the summation field of every component when one antenna is excited.

The impedance of radiation patch and feed network should be conjugate matched perfectly to obtain good efficiency of antenna. The I/O impedance of feed network shall be measured precisely and conjugate matched with the radiation patch. In this paper, double-feed $\mathrm{CP}$ antenna array is chosen to introduce the novel method of obtaining higher radiation efficiency.

This paper is organized as follows. In the second section, the radiation patch and feed network are introduced in detail. In the third section, theory analysis of axial ratio with mutual coupling effect is deduced. The novel method is applied in antenna array, thanks to which the performances of the antenna array will be improved. The last section will give the conclusion.

\section{Structure of the Dual-Feed Patch}

In accordance with the theory of the strip line, the character impedance of ideal strip line can be precisely calculated by the conformal mapping method. There is also some approximate arithmetic that can be adopted to shorten the calculation 


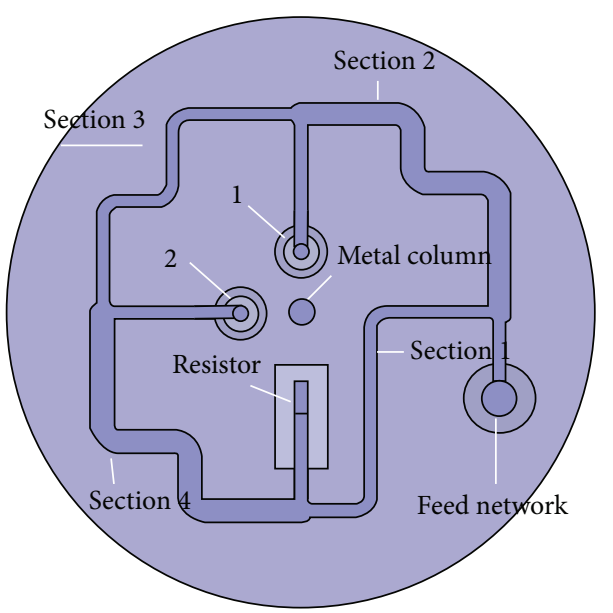

(a)

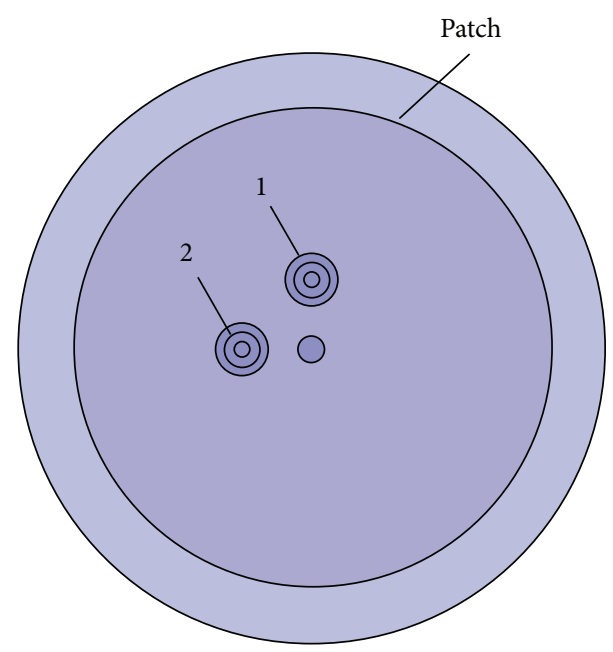

(b)

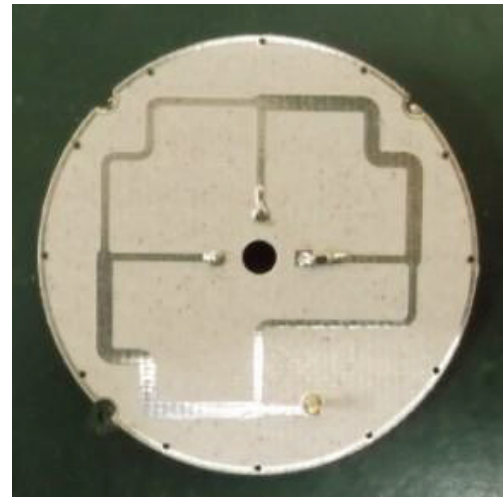

(c)

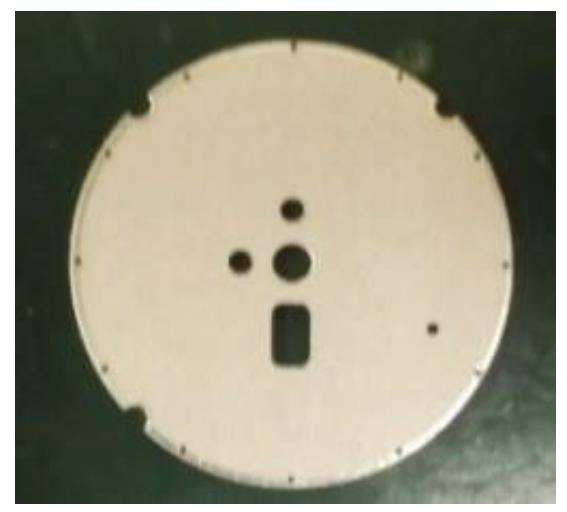

(d)

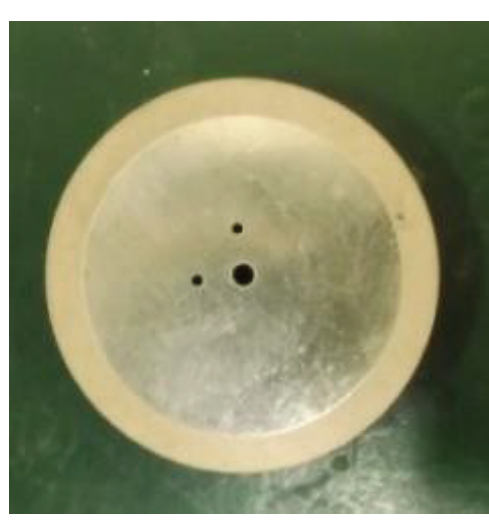

(e)

FIGURE 1: (a) shows the top view of the feed network which is a four-port strip line bridge, (b) is radiation patch on a thick PCB board of $4 \mathrm{~mm}$, (c) is bottom view of the feed network photo, (d) is the other half of the feed network, and (e) is the photo of radiation patch.

process. Actual processed strip line is always composed of two PCB layers which are tightly combined. As shown in Figures 1(a) and 1(b), there are models of the RHCP GPS dualfeed network and a patch which is operated at $1.575 \mathrm{GHz}$. The radius of the antenna is $17 \mathrm{~mm}$. The substrate permittivity of feed network is 4.6. The substrate permittivity of the radiation patch is 16 . The radius of the metal patch is $13.5 \mathrm{~mm}$. The feed network is a four-port electric bridge which contains one feed port, two output ports, and one isolation port. In Figure 1(a), 1 and 2 are the output ports which could supply equal amplitude and orthogonal signals for the patch in order to obtain the circular polarization. The isolation port is marked by resistor which can absorb leaking power and keep good performance of the power splitter. The feed network contains four sections of lines which have the same length equal to $23.4 \mathrm{~mm}$ or $1 / 4$ wavelength. The width of Sections 1 and 3 is the same $(1.304 \mathrm{~mm})$, so is the width of Sections 1 and $2(0.72 \mathrm{~mm})$. The thickness of the feed network and radiation patch is $1.62 \mathrm{~mm}$ and $4 \mathrm{~mm}$, respectively.

Figure 1(a) shows a model of four-port strip line bridge. Port 1 and 2 supply equal amplitude and orthogonal phase signals to the patch. In theory, the input and output impedance of a good bridge are $50 \mathrm{ohm}$, but the impedance of a patch is greatly affected by the mutual coupling when the antenna is located in array. The problem will be studied and solved in the next section. Strip line feed networks are the combination of two pieces of PCB board which are shown in Figures 1(c) and 1(d). Figure 1(e) shows the top view of radiation patch.

The alone antenna could obtain good performances for GPS application. But in the antenna array, the efficiency of the antenna will decrease because of mutual coupling. The paper proposes an effective method for solving this problem in the next section.

\section{Radiation Efficiency Improvement Method and Analysis}

The performances of the antenna radiation pattern and axial ratio can be deteriorated due to mutual coupling. The pattern of antenna array is analyzed in many papers whereas the axial ratio of that is rarely analyzed. In Section 3.1, the axial ratio theory analysis of circular polarization antenna array is deduced. In order to solve the problem, the radiation efficiency improvement method is proposed and analyzed in 
other sections. Seven-component antenna array is applied to show the effect of this novel method by experiments.

3.1. The Axial Ratio Analysis of Circular Polarization Antenna Array. The inner electronic field distribution of patch antenna is clearly known, so it is appropriate to be used to calculate the coupling efficiency which has been analyzed in many papers including radiation pattern and mutual impedance [10-13]. But the coupling effect study of axial ratio is rarely reported. In this section, the mutual coupling effects of axial ratio are deduced in theory. In circular polarization antenna, two resonant models have the same amplitude with 90- degree phase difference. The coupling effect of axial ratio is analyzed in theory by using two similar circular polarization microstrip antennas as shown in Figure 2. In Figure 2, axial ratio of antenna $A$ is 1 when antenna $B$ is not located beside it. Axial ratio is deduced when only antenna $\mathrm{A}$ is excited and antenna B is not excited. The analyzed conclusions of this model could be widely spread to various microstrip circular polarization antennas with different numbers and locations. The radius of these two antennas is marked by $a$.

Before obtaining the final axial ratio, the mutual reaction between antennas should be calculated. Similar to the mutual reaction in the calculation of dipole mutual impedance, the mutual reaction is defined as

$$
\langle a, b\rangle=-\iint_{s_{b}} \vec{H}_{a b} \cdot \vec{M}_{b} d s,
$$

where $s_{b}$ is the area occupied by the source $\vec{M}_{b}$ and $\vec{H}_{a b}$ is the magnetic field produced by the current $\vec{M}_{a}$ on the aperture of patch $\mathrm{B}$. The $\vec{H}_{a b}$ is obtained from the electric vector potential $\vec{F}_{a}$ using

$$
\vec{H}_{a b}=\frac{1}{j \omega \mu}\left[k_{0}^{2} \vec{F}_{a}+\nabla\left(\nabla \cdot \vec{F}_{a}\right)\right],
$$

where $\vec{F}_{a}$ is given by

$$
\begin{gathered}
\vec{F}_{a}=\iint_{s_{a}} \overline{\bar{G}}\left(r, r^{\prime}\right) \cdot \vec{M}_{a} d s \\
\overline{\bar{G}}\left(r, r^{\prime}\right)=\left[\overline{\bar{I}}+\frac{1}{k_{0}^{2}} \nabla \nabla\right] G_{0}\left(r, r^{\prime}\right) .
\end{gathered}
$$

Here, $S_{a}$ is the area of the magnetic current of antenna A. $\overline{\bar{G}}\left(r, r^{\prime}\right)$ is the free space dyadic Green function. However, for low values of permittivity and small substrate thickness, conditions which are usually true for microstrip antennas, one can use the free space Green function with negligible error. The electric field distribution for the $T M_{m n}$ mode inside the patch metallization is given by

$$
E(\rho, \phi)=A J_{n}\left(k_{m n} \rho\right) \cos n \phi \vec{z} .
$$

The equivalent magnetic surface current at the border of antenna $\mathrm{A}$ is shown as in (6) and the magnetic current of other borders could be calculated by this equation:

$$
\vec{M}_{a}=2 \vec{E} \times \vec{n}=2 E_{z}(a, \phi) \vec{\phi}
$$

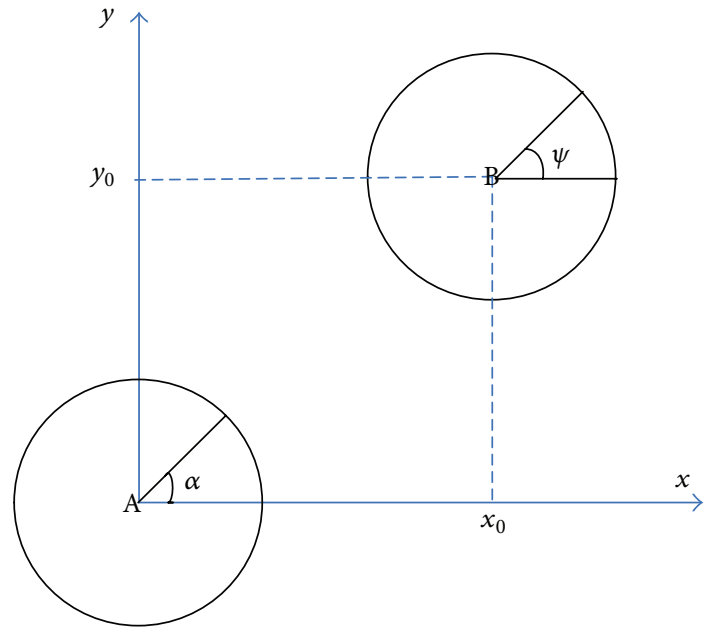

FIGURE 2: Diagram of the circular polarization antenna array which contains two microstrip antennas.

After obtaining the exact value of magnetic current around the periphery of antenna A, the magnetic field at xoy plane could be calculated by (2) and (3). The expressions for the components of $H_{a b}$ are obtained as

$$
\begin{aligned}
H_{a b}^{x}=\frac{1}{j \omega \mu} \int_{0}^{2 \pi} & \frac{a M_{a} \cos \alpha \cdot e^{-j k r}}{4 \pi r^{3}} \\
\cdot & \left\{2 \sin \alpha(1+j k r)+\frac{(y \sin \alpha+x \cos \alpha-a)}{r^{2}}\right. \\
& \left.\times\left(k^{2} r^{2}-3 j k r-3\right)\right\} d \alpha,
\end{aligned}
$$$$
H_{a b}^{y}=\frac{1}{j \omega \mu} \int_{0}^{2 \pi} \frac{a M_{a} \cos \alpha \cdot e^{-j k r}}{4 \pi r^{3}}
$$

$$
\begin{aligned}
& \cdot\left\{2 \cos \alpha(1+j k r)+\frac{(y \sin \alpha+x \cos \alpha)}{r^{2}}\right. \\
& \left.\times\left(k^{2} r^{2}-3 j k r-3\right)\right\} d \alpha,
\end{aligned}
$$

where $r^{2}=(x-a \cos \alpha)^{2}+(y-a \sin \alpha)^{2}$. The mutual react formulation could be derived below. Here, the value of $\vec{M}_{a}$ equals $\vec{M}_{b}$, whereas the locations of the two are different:

$$
\begin{aligned}
& \langle a, b\rangle_{y}=\int_{0}^{2 \pi} 2 E_{b}(a, \psi) H_{a b}^{y} \cos \psi d \psi . \\
& \langle a, b\rangle_{x}=\int_{0}^{2 \pi} 2 E_{b}(a, \psi) H_{a b}^{x} \sin \psi d \psi . \\
& \langle a, a\rangle_{y}=\int_{0}^{2 \pi} 2 E_{a}(a, \alpha) H_{a b}^{y} \cos \alpha d \alpha . \\
& \langle a, a\rangle_{x}=\int_{0}^{2 \pi} 2 E_{a}(a, \alpha) H_{a b}^{x} \sin \alpha d \alpha .
\end{aligned}
$$


This completes the derivation of mutual reaction between two identical circular microstrip patch antennas. The general field is the summation of the two patch antennas radiation. Using this expression, the axial ratio at the normal orientation could be deduced in

$$
\begin{aligned}
\operatorname{AR}=\frac{\max }{\min }[ & \left(\int_{0}^{2 \pi} e^{j(\pi / 2)} E_{a}(a, \alpha) H_{a b}^{y} \cos \alpha d \alpha\right. \\
& \left.+\int_{0}^{2 \pi} e^{j(\pi / 2)} E_{b}(a, \psi) H_{a b}^{y} \cos \psi d \psi\right)^{2} \\
& +\left(\int_{0}^{2 \pi} E_{a}(a, \alpha) H_{a b}^{x} \sin \alpha d \alpha\right. \\
& \left.\left.+\int_{0}^{2 \pi} E_{b}(a, \psi) H_{a b}^{x} \sin \psi d \psi\right)^{2}\right]^{(1 / 2)}
\end{aligned}
$$

The axial ratio is deduced when another antenna is located beside excited antenna $\mathrm{A}$. It is clear that the axial ratio could also be obtained when any other antennas are located beside one excited circular polarization antenna. Therefore, the input impedance, radiation pattern, and axis ratio are simulated and measured in detail in this section and the next couple of sections.

3.2. Impedance Analysis of Antenna Feed Point. In order to lessen the negative effect of mutual coupling, the detailed impedance analysis of the patch is of great importance. The input impedance of the patch is closely related to the location of the feed point [10]. The input resistance of the circular microstrip patch could be calculated by the following equations when the antenna is operated at the main resonant mode:

$$
R=\frac{V^{2} J_{1}^{2}\left(k \rho_{0}\right)}{P_{T}},
$$

where $V$ is the voltage which is the integral of electric field $E$ through height $h . J_{1}\left(k \rho_{0}\right)$ is the one-order Bessel function. $\rho_{0}$ is the distance at radius, $k$ is the propagation constant, and $P_{T}$ is the consumption power which include the radiation power, consumption on the metal, and the substrate. If $\rho_{0}$ becomes smaller, $J_{1}\left(k \rho_{0}\right)$ and $V$ will also be smaller, and visa verse. In (10), $\rho_{0}$ is the variable. Simulation results of the antenna of Figure 1(b) are shown in Figure 3 which is the relationship between the input impedance and $\rho_{0}$.

In Figure 3, the resistance is so high when the antenna operates at the resonant frequency. But if the antenna does not operate at the resonant frequency, the reactance value shall not be 0 . In this situation, the patch should be conjugate matched with the feed network in order to obtain enough efficiency. The reflection between the two is very large when their reactance properties are the same. In this paper, the theory is applied to obtain good effect on the improvement of the antenna gain. Just like Section 3.1, in circularity polarization antenna array, the resonant resistances of two polarizations in one antenna are affected by coupling differently, so the matching and axial ratio will be worse.

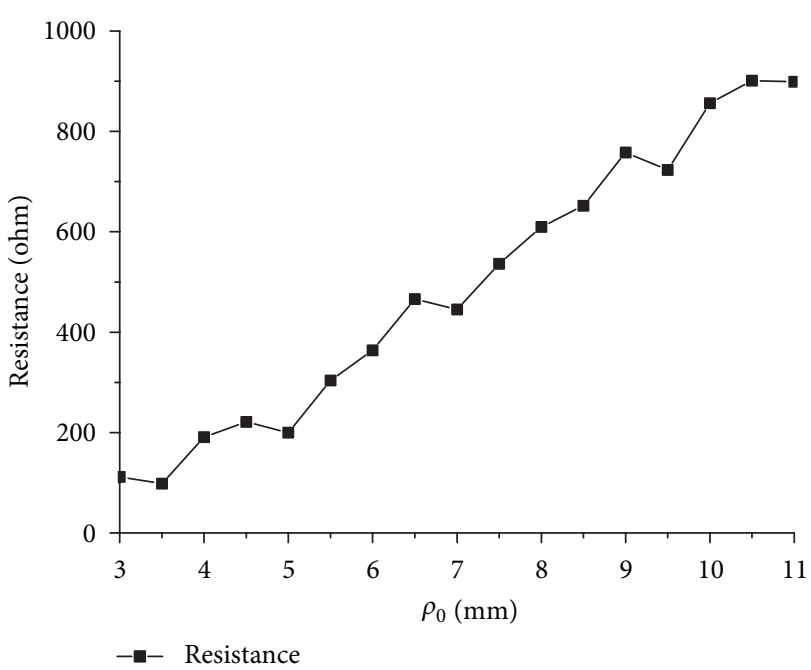

FIGURE 3: Resonant resistance versus different feed point.

3.3. Radiation Efficiency Improvement Method. After analyzing the relationship between feed point and impedance, seven-component circularity polarization antenna array is applied as the example in order to establish experiments of improvement method in this section. Figure 4 is the overview of seven-component antenna arrays which are of hexagonal shape and widely applied in the antijam navigation system. In order to investigate the performances of mutual coupling well, the antenna model in Figure 4(a) contains seven patches as in Figure 1(b). Figure 4(b) shows the seven integrated antennas which contain feed network and patch. Figure 4(a) has 14 ports, whereas Figure 4(b) has 7 ports. The impedance of each patch in Figure 4(a) also can be measured as follows: the target antenna is connected to vector network analyzer, while other antennas and ports are connected with matched load.

In Figure 4(a), feed points of each component are arranged in parallel. The distance among the adjacent component is $68 \mathrm{~mm}$ which is of 0.35 wave length. The array contains only seven components, so that each component in this array operates at different situations. It is easy to understand that each port is barely affected by other ports which operate at the orthogonal mode. Therefore, the working status of each port is mainly affected by the other six ports which radiate the same polarization wave. The ports impedance of antenna 1 could be calculated by the self-impedance and the mutual impedance:

$$
\begin{aligned}
& Z_{a 1}=Z_{a 11}+Z_{a 21}+Z_{a 31}+Z_{a 41}+Z_{a 51}+Z_{a 61}+Z_{a 71} \\
& Z_{b 1}=Z_{b 11}+Z_{b 21}+Z_{b 31}+Z_{b 41}+Z_{b 51}+Z_{b 61}+Z_{b 71}
\end{aligned}
$$

where subscripts $a$ and $b$ are ports 1 and 2 of each antenna, respectively. $Z_{b 1}$ is the input impedance of antenna 1's port $2, Z_{b 11}$ is the self-impedance of antenna 1's port 2, and $Z_{b i 1}$ is the mutual impedance between port 2 of antenna $i$ and that of antenna 1. From Figure 4(a), it could be easily deduced that $Z_{a 11}=Z_{b 11}$ and $Z_{a 21} \neq Z_{b 21}$. Therefore, the two resonant modes are affected by coupling differently and $Z_{a 1} \neq Z_{b 1}$. The 


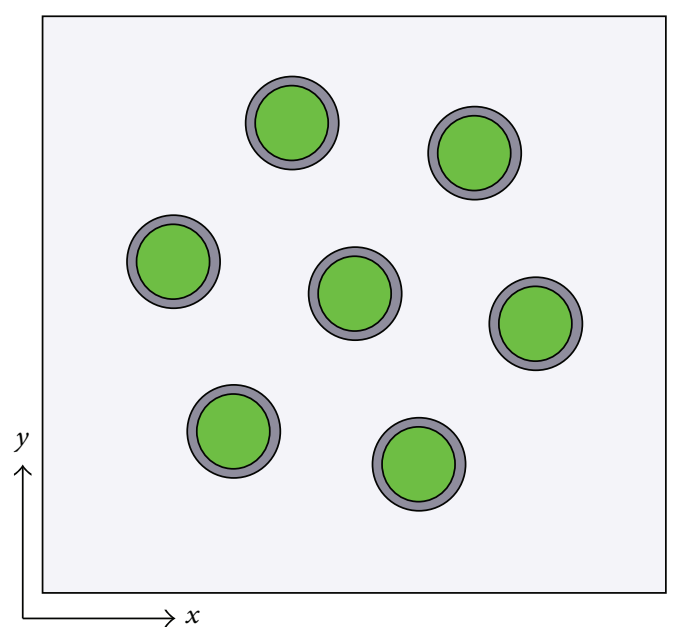

(a)

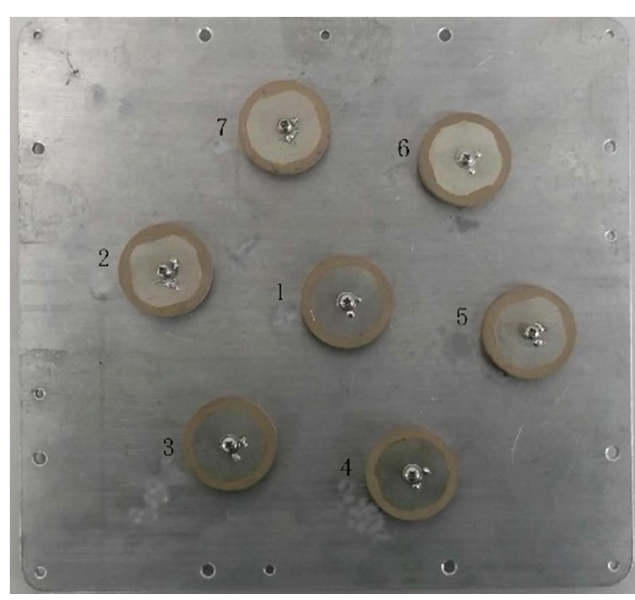

(b)

FIGURE 4: Seven-component antenna array: (a) a model without feed network and (b) final product of array.

TABLE 1: Measured input impedance of each port.

\begin{tabular}{lccccccc}
\hline Port & $a 1$ & $a 2$ & $a 3$ & $a 4$ & $a 5$ & $a 6$ & $a 7$ \\
Impedance & $132+17 * j$ & $75-33 * j$ & $78-7 * j$ & $77-29 * j$ & $66-16 * j$ & $75-11 * j$ & $78-12 * j$ \\
\hline Port & $b 1$ & $b 2$ & $b 3$ & $b 4$ & $b 5$ & $b 6$ & $b 7$ \\
Impedance & $105-12 * j$ & $67+6 * j$ & $84-26 * j$ & $74-29 * j$ & $46+7 * j$ & $68-37 * j$ & $76-32 * j$ \\
\hline
\end{tabular}

radiation perturbation is caused by the other antennas which can be excited by coupling. The measured impedance of each port in the antenna array of Figure 4 at $1.575 \mathrm{GHz}$ is presented in Table 1.

In Table 1, the input impedance of each port in the antenna array is presented. Measured input impedance of each port is $69-9 * j$ when the antenna stays alone. Mismatch leads to unequal power and distorted phase delay between the two ports of antenna. The paper proposes a novel experimental method to solve the problem of mismatch. Through applying this method, the efficiency of each component could be improved significantly.

The antenna could be designed as in Figure 5(a). The stubs of the antenna are applied for adjusting of the input impedance. By adjusting the length of two opposing stubs, the experiment could change the input impedance of each port, because the input impedance of microstrip antenna is mainly decided by the location of feed point. The measured port 1 and port 2 of the feed network are $51+15 * j$ and $48+23 * j$, respectively. The best patch impedance of ports 1 and 2 should approach $51-15 * j$ and $48-23 * j$, respectively. Figure 5(b) shows the Smith chart of number 2 antenna which is fed at port 1 . If the stubs become shorter, the marker of any frequency will rotate around the curve along the anticlockwise orientation. But there is huge difference between stubs 1 and 2 . The feed point effectively moves to edge if stub 1 becomes shorter; thus, the input impedance will become larger and the curve in Smith chart will move to the right, and vice versa. If stub 2 becomes shorter, the curve will entirely move to the left. So, this is a very important rule for changing the impedance experimentally. By adjusting the stubs' length, the changed impedances of all ports are shown in Table 2. The dimensions of improved antenna stubs are shown in Table 3. For example, "11" means the length of stub 1 of number 1 antenna. The width of all stubs is 1 centimeter.

3.4. Measurement Results Analysis of the Novel Method. After improving the impedance of each antenna, the efficiency of each antenna is improved in various degrees. The pattern of original and improved antenna should be measured at the same place and same time, in order to obtain believable results. In Figure 6, the measurement scenario is shown; every component of the antenna array operates independently. Figure 6(a) shows the photo of transmitter antenna which is fixed on the vertical rotatable platform. Figure 6(b) shows that the receiver antenna array is fixed on the platform which can rotate at both vertical and horizontal orientation. The radiation pattern and axial ratio could be easily measured. Figure 7(a) shows the S11 performance of single antenna which is the combination of patch and feed netword. The $-10 \mathrm{~dB}$ bandwidth is much larger than $100 \mathrm{MHz}$ because one resistor locates at the isolation port in feed network to keep balance. In this paper, the S11 performance of the integrated antenna is not discussed in detail because it is always good during the experiment. Figure 7(b) shows the gain performance when the antenna stays alone. The gain is the largest when the antenna operates at $1.575 \mathrm{GHz}$. The 


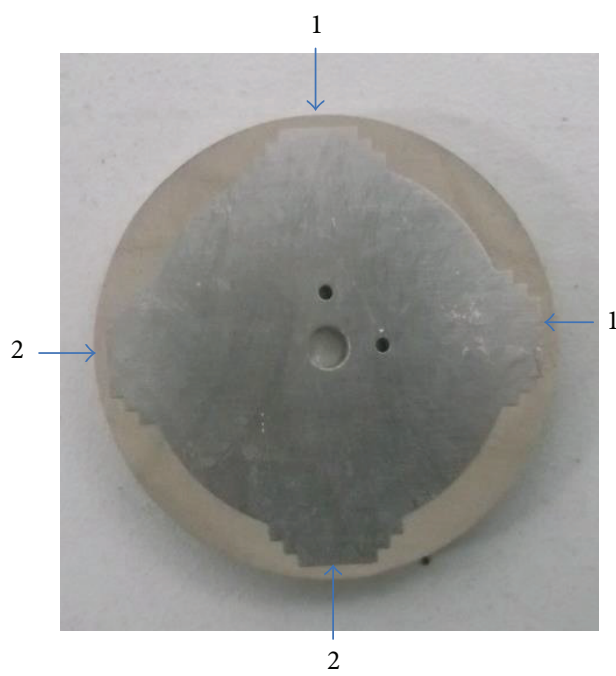

(a)

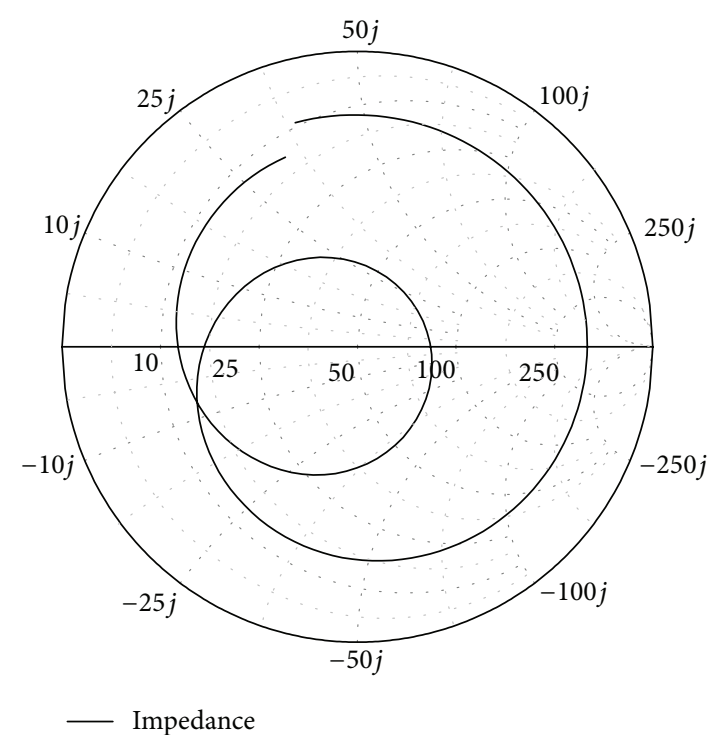

(b)

Figure 5: (a) The photo of improved antenna which contains two couples of stubs. (b) Measured number 2 component Smith chart versus frequency.

TABLE 2: Measured improved input impedance of each port.

\begin{tabular}{lccccccc}
\hline Port & $a 1$ & $a 2$ & $a 3$ & $a 4$ & $a 5$ & $a 6$ & $a 7$ \\
Impedance & $52-17 * j$ & $48-23 * j$ & $53-21 * j$ & $61-19 * j$ & $56-16 * j$ & $51-21 * j$ & $56-28 * j$ \\
\hline Port & $b 1$ & $b 2$ & $b 3$ & $b 4$ & $b 5$ & $b 6$ & $b 7$ \\
Impedance & $55-6 * j$ & $53-16 * j$ & $54-27 * j$ & $51-22 * j$ & $55-17 * j$ & $56-29 * j$ & $59-22 * j$ \\
\hline
\end{tabular}

TABLE 3: Dimensions of the improved antennas.

\begin{tabular}{lccccccc}
\hline Stub & 11 & 21 & 31 & 41 & 51 & 61 & 71 \\
Length $(\mathrm{mm})$ & 0.3 & 0.3 & 0.1 & 0.1 & 0.1 & 0 & 0.1 \\
\hline Stub & 12 & 22 & 32 & 42 & 52 & 62 & 72 \\
Length $(\mathrm{mm})$ & 0.2 & 0 & -0.1 & -0.2 & 0 & 0.2 & 0.3 \\
\hline
\end{tabular}

gain will decrease when the operating frequency of antenna is deviated. The novel method is mainly applied in the single frequency point, so that the gain performances of the resonant frequency are discussed in detail. Figure 7 (c) shows the efficiency performance with different frequency.

In Figures 8(a), 8(b), and 8(c), the comparison between the original and the improved pattern of three antennas is demonstrated. Number 2, number 4, and number 6 in antenna array of Figure 4(b) are selected as an example. The picture shows the normalized power radiation pattern which is the power composition of the vertical and horizontal electrical field part. All the patterns are measured at xoz plane and normalized by the original gain. In Figure 8(d), the gain difference between original antenna array and improved antenna array is shown. It is clear that the gain of the improved antenna array is increased. Because the shapes of patterns are similar between original antenna and improved antenna, the gain difference approximately equals efficiency difference.

In Figure 8, it could be easily observed that, after adopting novel technology, the efficiency of all antennas is improved. It is proved that the novel technology is of enormous use in lessening the negative effect which results in mutual coupling. Mutual coupling leads to the differences among the pattern shapes of antennas. The pattern shape of each antenna is not changed significantly after improvement because the pattern is relatively robust. The radiation pattern is determined by not only the location of the component but also the mutual coupling between components. In induction theorem, electromagnetic wave will be received by other components and radiated again when only one component is excited. The final radiation pattern is the superposition of the radiation of every component. The axial ratio is not robust and improved subtlety when the novel technology is applied. In order to study the axial ratio performance further, the axial ratio performances of the same antennas are also shown in Figure 9.

Figure 9 selects three antennas and shows comparison of axial ratio between the original antennas and the improved ones. It is observed that the axial ratio is improved when the novel method is applied; axial ratios of these antennas are all lower than $3 \mathrm{~dB}$ at the axial orientation. The axial ratio is easily changed by coupling of other components, especially 


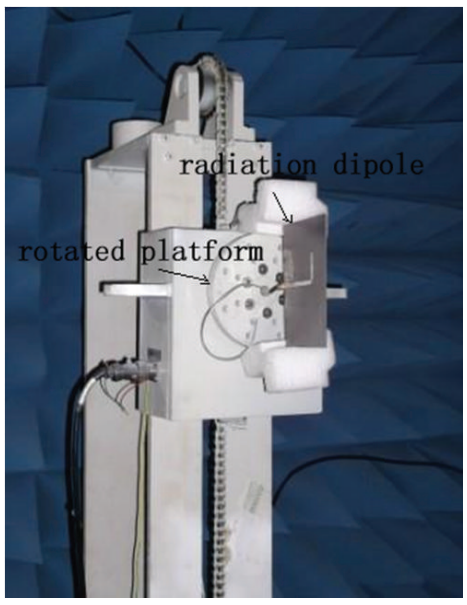

(a)

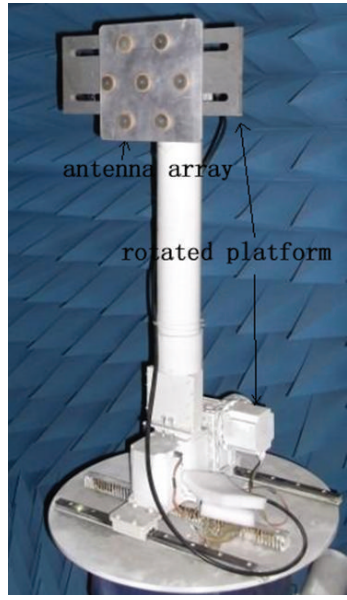

(b)

FIGURE 6: Measurement scenarios: (a) radiation antenna is fixed on the rotatable platform. (b) Received antenna is fixed on rotatable platform.

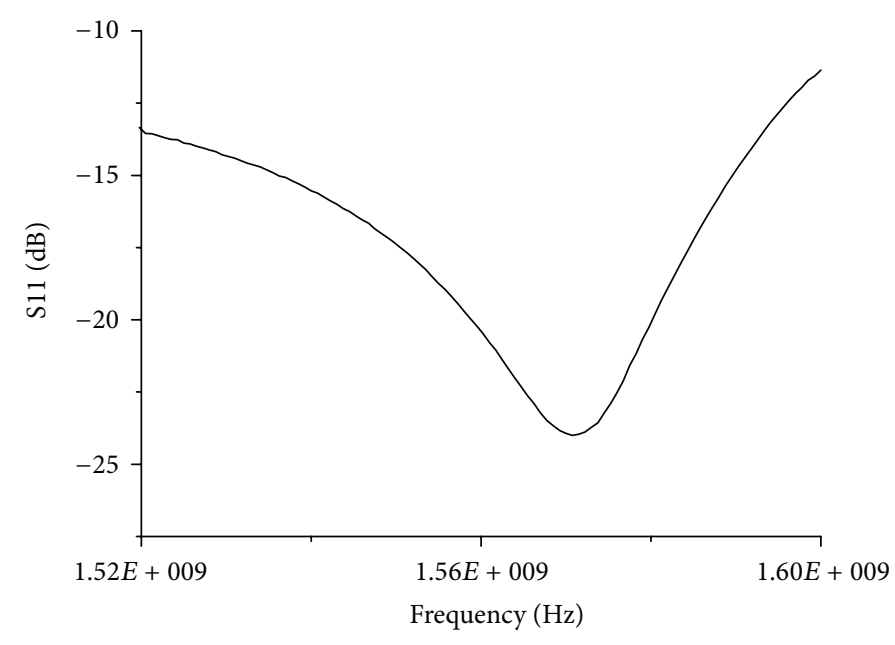

S11

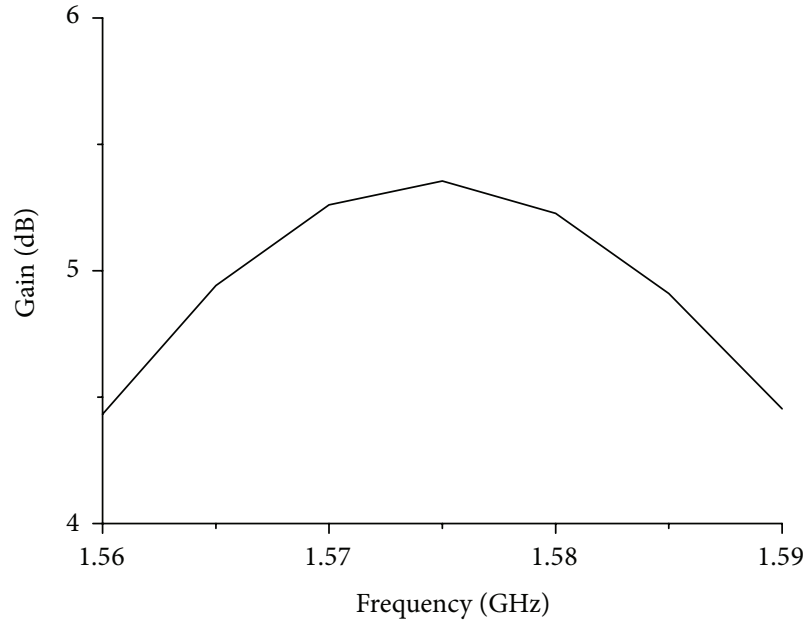

(b)

(a)

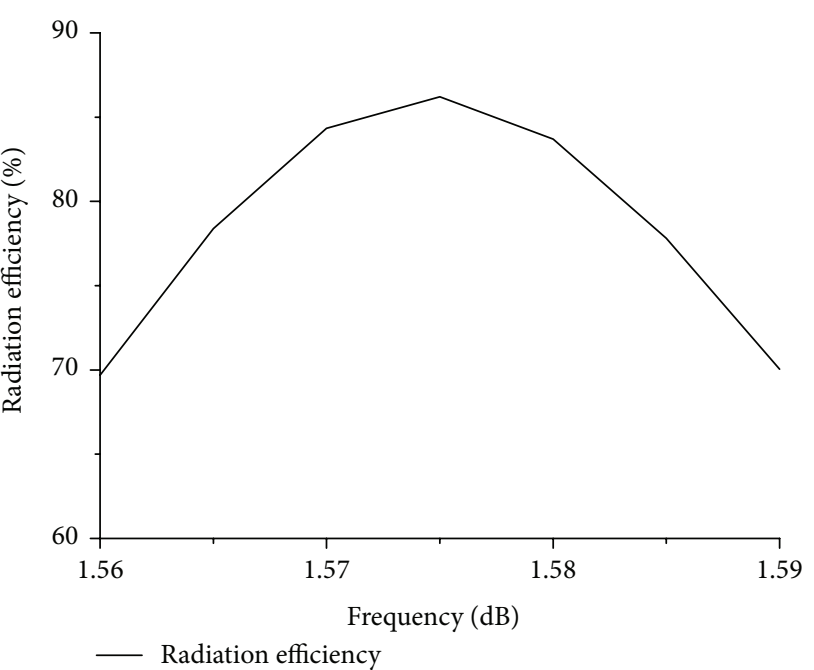

(c)

Figure 7: Performances of integrated single antenna. (a) S11, (b) gain, and (c) efficiency. 


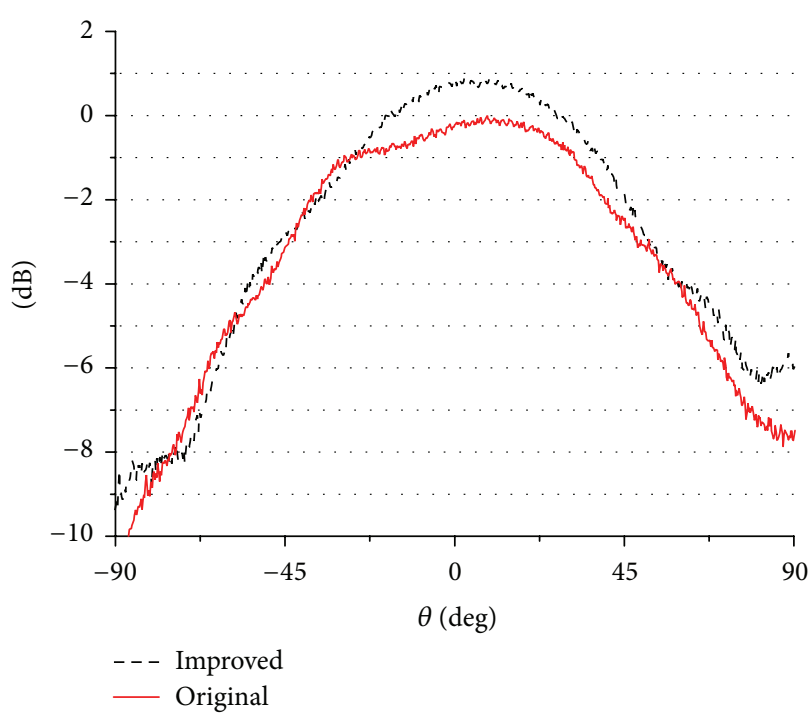

(a)

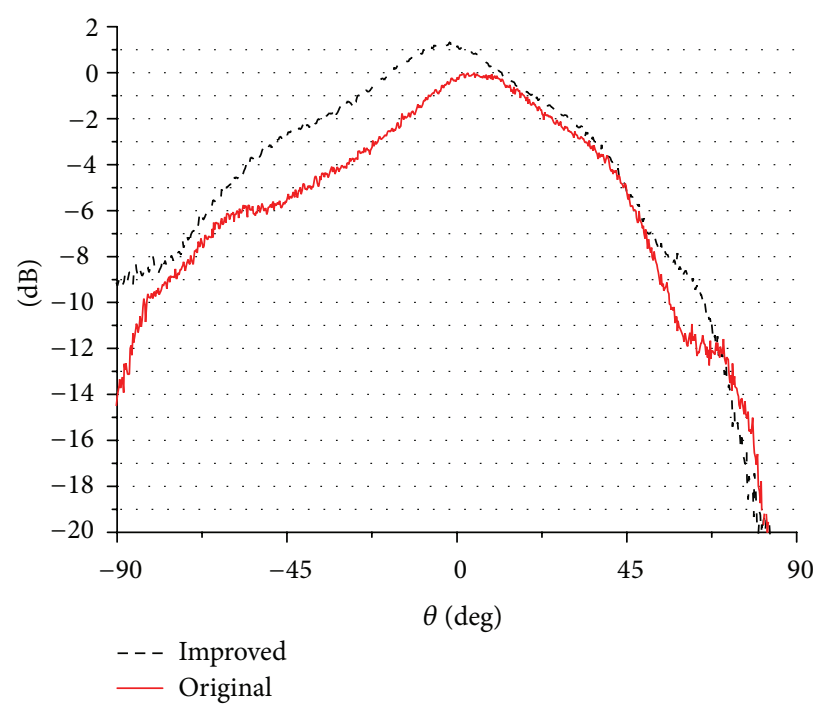

(c)

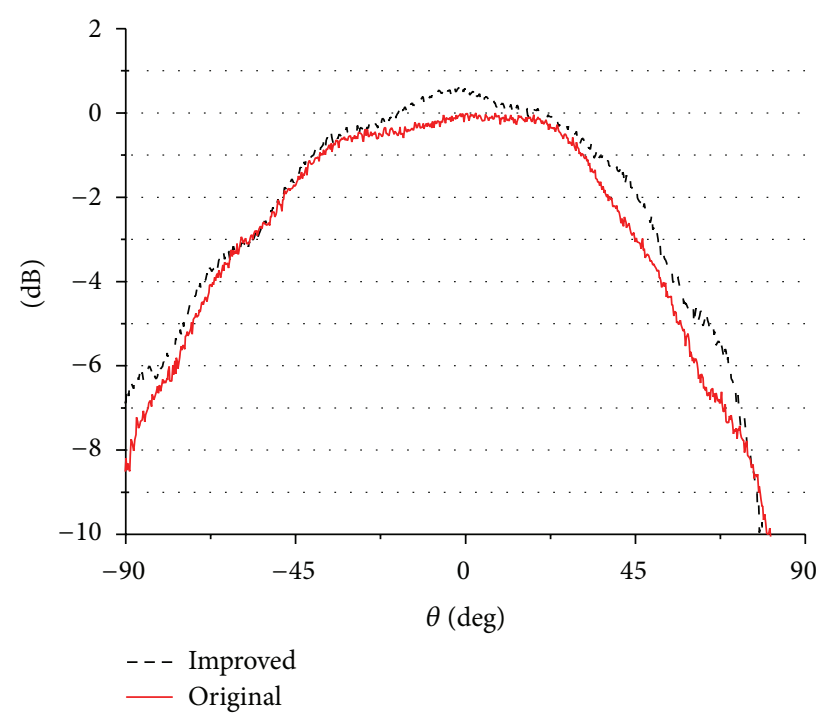

(b)

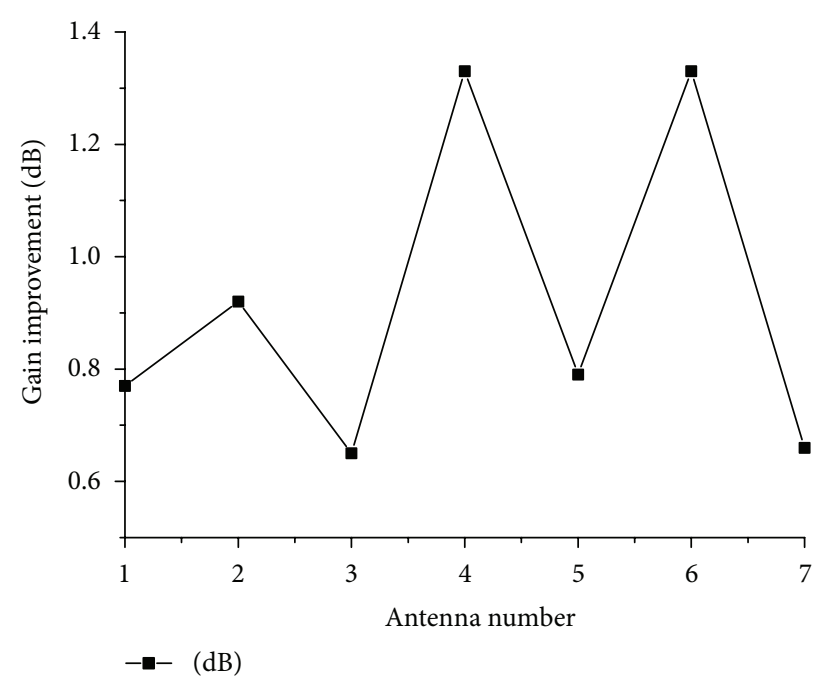

(d)

FIGURE 8: Comparing between improved pattern and original pattern: (a) number 2 component, (b) number 4 component, (c) number 6 component, and (d) efficiency/gain improvement curve.

in the low elevation. The distortion of radiation pattern also influences the axial ratio because the beam fails to point to the normal orientation. Therefore, the axial ratio is worse than before at some directions, which is very hard to be avoided. The novel method is applied to improve the match and raise the radiation efficiency of circular polarization antenna. Actually, the AR of axial orientation is improved under most of the conditions. Especially, the axial ratio performance at normal direction is the most important one for the circular polarization antenna.

\section{Conclusion}

In this paper, one novel debugging method aiming at improving the efficiency of circular polarization antenna in array was proposed. By introducing the stubs and using the Smith chart, the impedance of each patch could be well conjugate matched with the feed network. Owing to the well matched antenna patch constructed, the radiation efficiency could be increased by about $1 \mathrm{~dB}$. Due to their good characteristic of increasing efficiency, the circularity polarization multifeed antennas in small antenna array could obtain weaker signal from planet and earn better navigation effect.

Moreover, the axial ratio in antenna array is also numerically analyzed and easily distorted by various electromagnetic circumstances. In antenna array, the main causation is that other antennas will be excited by mutual coupling.

\section{Conflict of Interests}

The authors declare that there is no conflict of interests regarding the publication of this paper. 


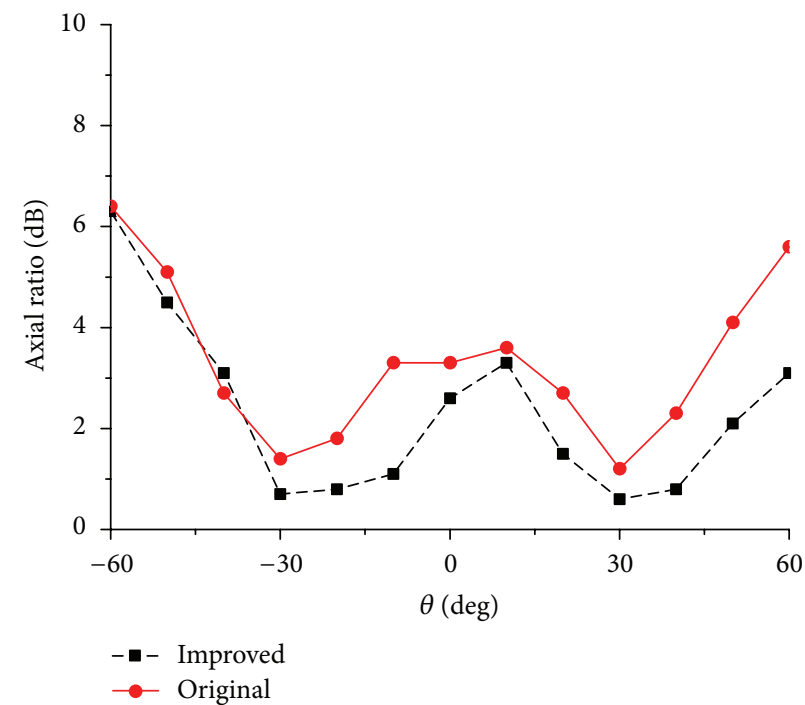

(a)

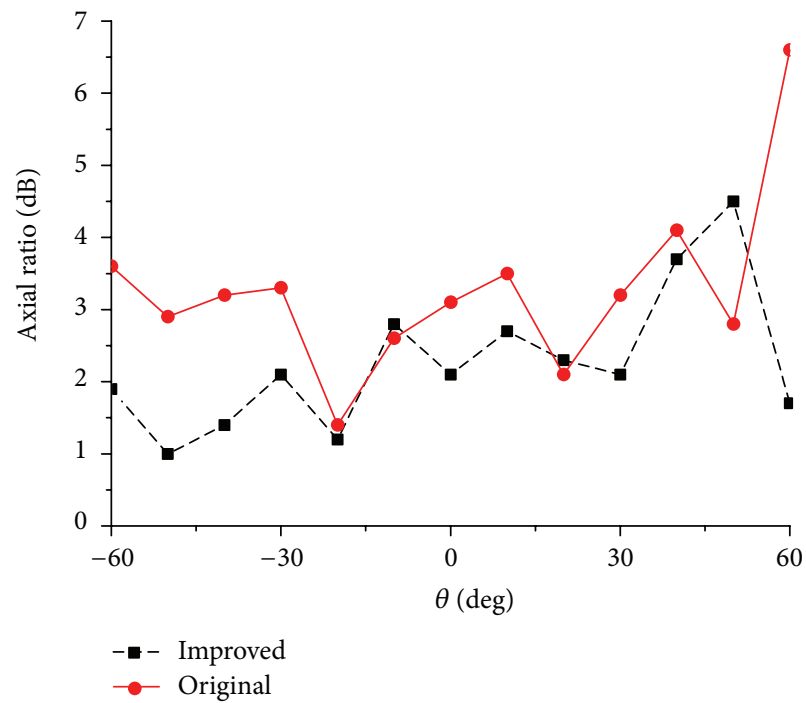

(b)

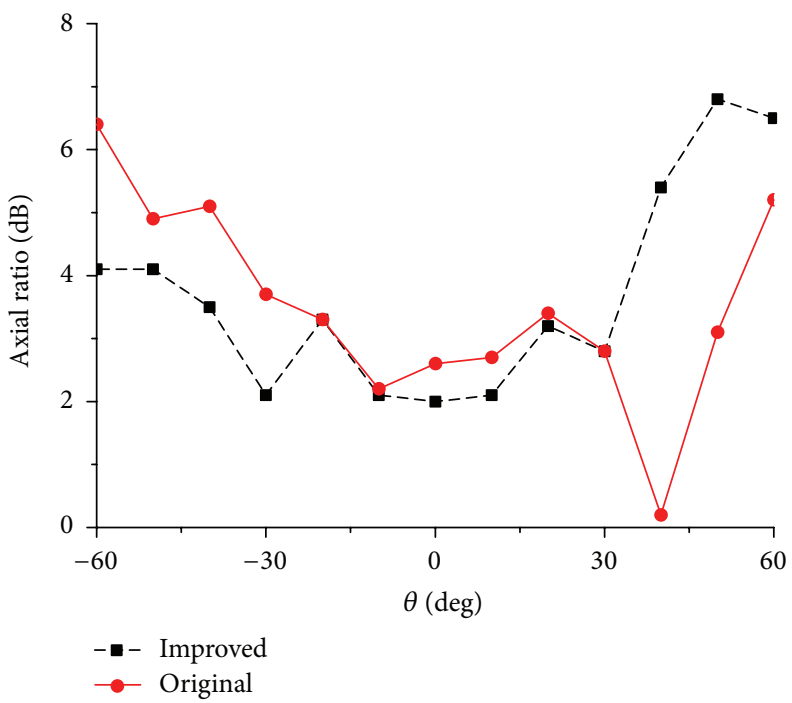

(c)

Figure 9: Axial ratio performances of the three components: (a) number 2 component, (b) number 4 component, and (c) number 6 component.

\section{Acknowledgments}

This work was supported in part by the National Natural Science Foundation of China (nos. 61301093 and 61271416) and the National Defense Pre-Research Foundation of China (no. 11055132535).

\section{References}

[1] A. H. Sharif and M. Soleimani, "Accurate analysis and design of dual-feed circularly polarized microstrip patch antenna based on multiport network model," in Proceedings of the Asia-Pacific Microwave Conference (APMC '05), vol. 4, December 2005.

[2] R. D. Murch and K. Ben Letaief, "Antenna systems for broadband wireless access," IEEE Communications Magazine, vol. 40, no. 4 , pp. $76-83,2002$.
[3] Y. Ushijima, E. Nishiyama, and M. Aikawa, "Single-layer integrated microstrip array antenna for dual circular polarisation microwaves," IET Microwaves, Antennas \& Propagation, vol. 6, no. 8, pp. 962-968, 2012.

[4] J. Ouyang, "A circularly polarized switched-beam antenna array," IEEE Antennas and Wireless Propagation Letters, vol. 10, pp. 1325-1328, 2011.

[5] W.-T. Hsieh, T.-H. Chang, and J.-F. Kiang, "Dual-band circularly polarized cavity-backed annular slot antenna for GPS receiver," IEEE Transactions on Antennas and Propagation, vol. 60, no. 4, pp. 2076-2080, 2012.

[6] Y.-J. Hu, W.-P. Ding, and W.-Q. Cao, "Broadband circularly polarized microstrip antenna array using sequentially rotated technique," IEEE Antennas and Wireless Propagation Letters, vol. 10, pp. 1358-11361, 2011. 
[7] A. D. Nesic and D. A. Nesic, "Printed planar $8 \times 8$ array antenna with circular polarization for millimeter-wave application," IEEE Antennas and Wireless Propagation Letters, vol. 11, pp. 744747, 2012.

[8] C. Vázquez, G. R. Hotopan, S. ver Hoeye, M. Fernández, L. F. Herrán, and F. las Heras, "Microstrip antenna design based on stacked patches for reconfigurable two dimensional planar array topologies," Progress in Electromagnetics Research, vol. 97, pp. 95-104, 2009.

[9] P. Rocca, L. Manica, and A. Massa, "An effective excitation matching method for the synthesis of optimal compromises between sum and difference patterns in planar arrays," Progress in Electromagnetic Research B, vol. 3, pp. 115-130, 2008.

[10] I. J. Bahl and P. Bhatia, Microstrip Antenna, Publishing House of Electronics Industry, 1984.

[11] D. M. Pozar, "Input impedance and mutual coupling of rectangular microstrip antennas," IEEE Transactions on Antennas and Propagation, vol. 30, no. 6, pp. 1191-1200, 1982.

[12] C. H. Niow, Y. T. Yu, and H. T. Hui, "Compensate for the coupled radiation patterns of compact transmitting antenna arrays," IET Microwaves, Antennas and Propagation, vol. 5, no. 6, pp. 699704, 2011.

[13] S. R. Best, "Mutual coupling between orthogonal electrically small dipole antennas," in Proceedings of the 6th European Conference on Antennas and Propagation (EUCAP '12), pp. 1663-1666, 2012. 

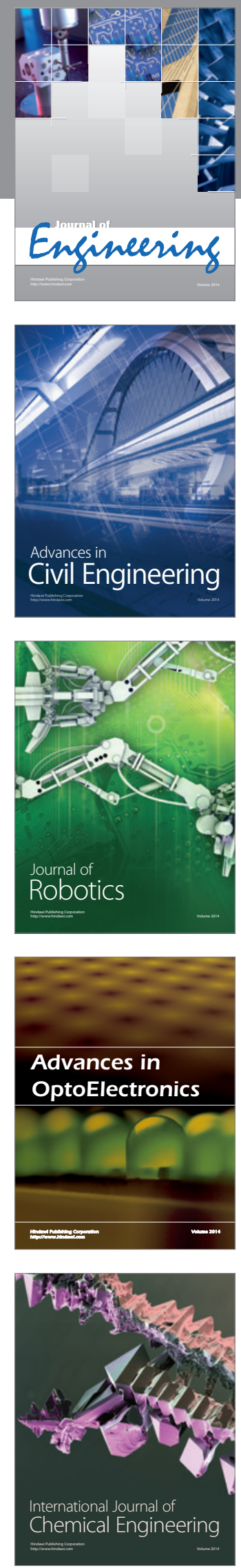

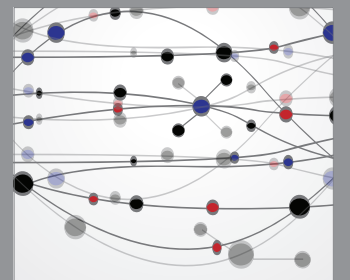

The Scientific World Journal
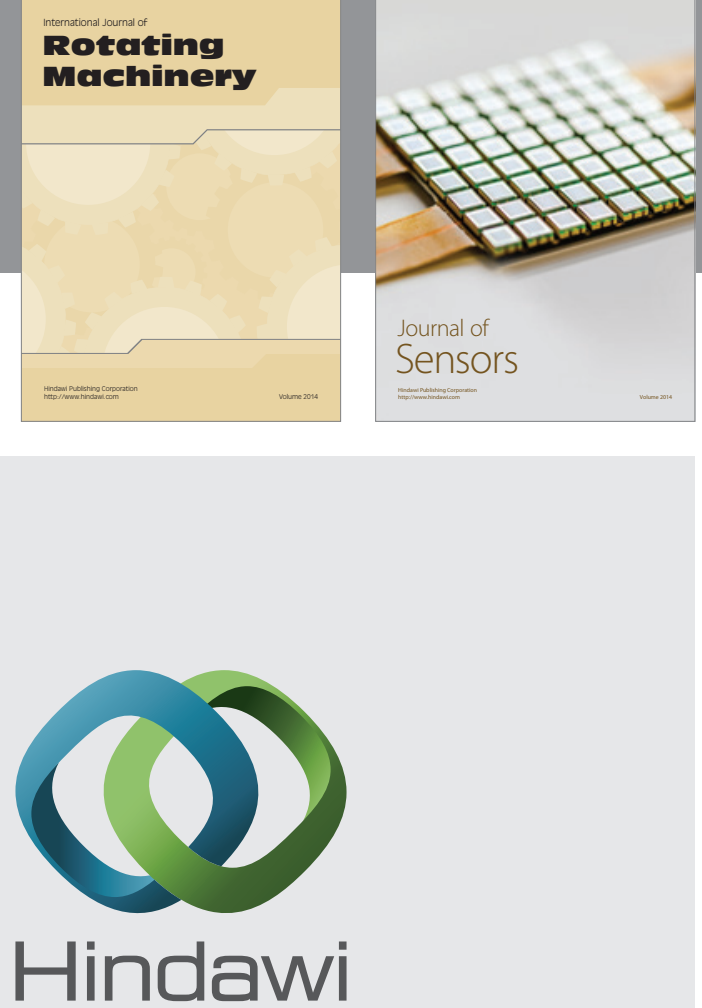

Submit your manuscripts at http://www.hindawi.com
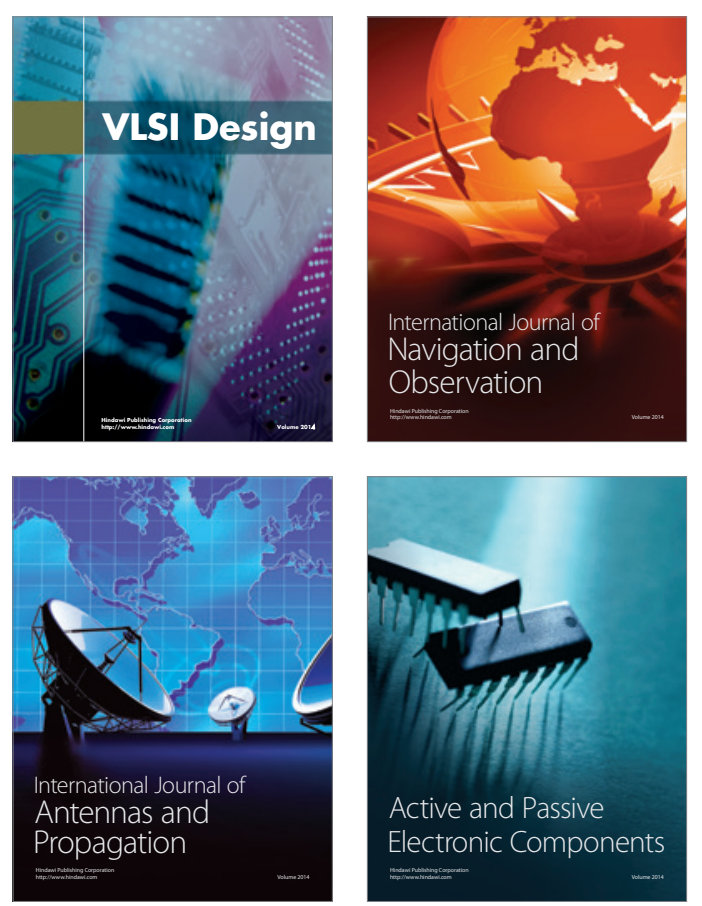
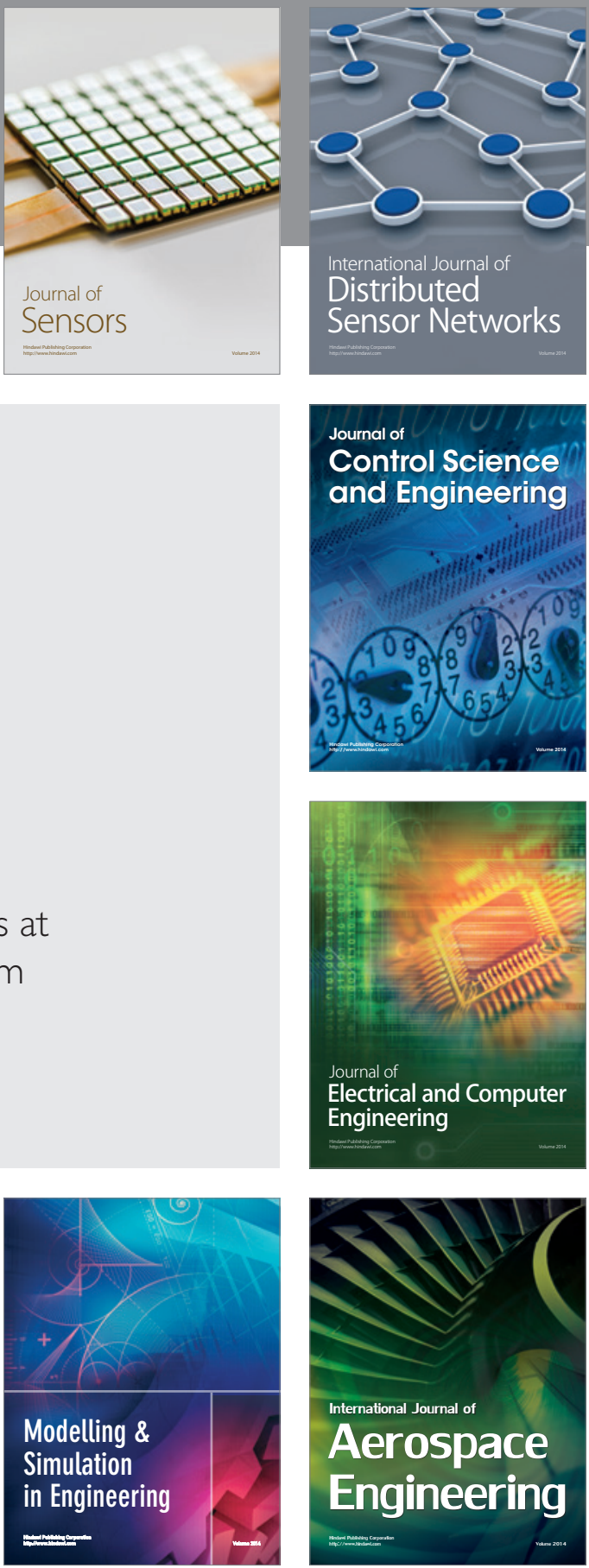

Journal of

Control Science

and Engineering
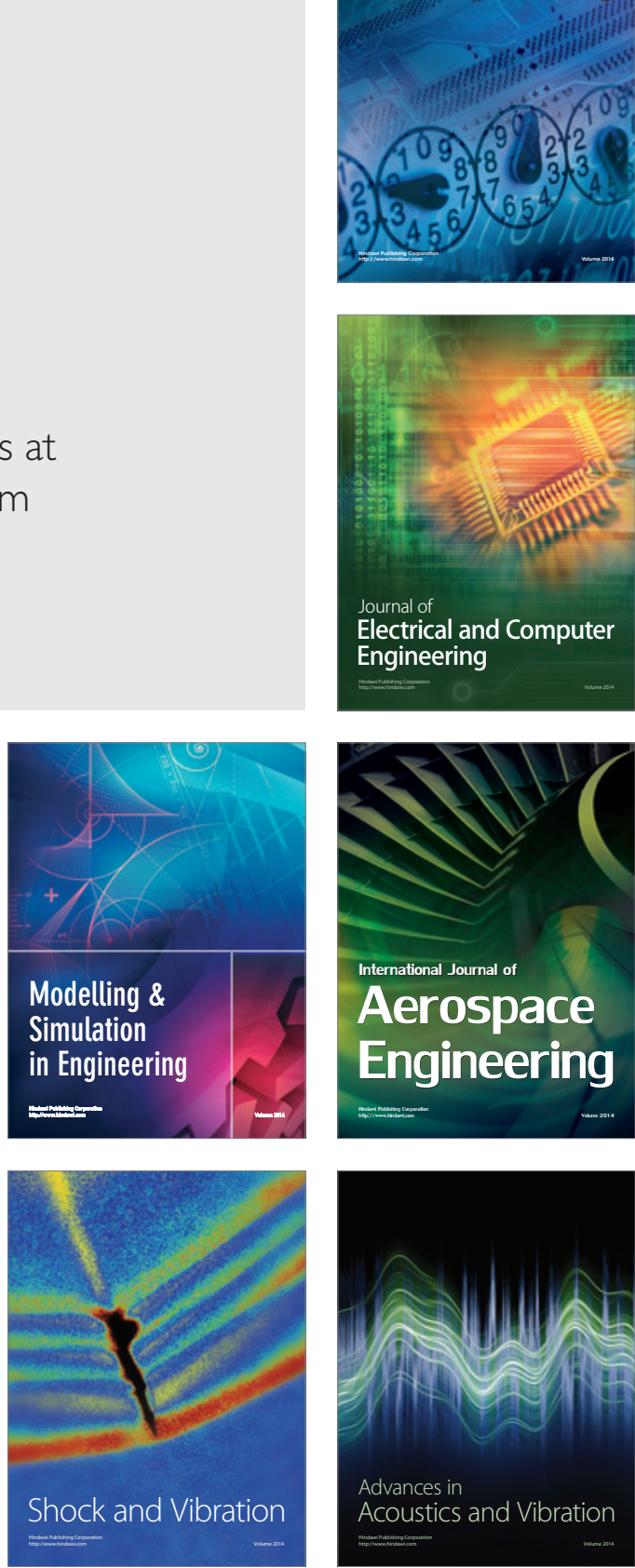